\title{
Impact of COVID-19 pandemic on plastic surgery activities and residency programs in a tertiary referral centre in Iran
}

\author{
Abdoljalil Kalantar-Hormozi ${ }^{1}$ - $\cdot$ Zahra Habibzadeh $^{2} \cdot$ Masoud Yavari $^{2} \cdot$ Seyed Mehdi Mousavizadeh $^{2} \cdot$ \\ Seyed Esmaeil Hassanpour ${ }^{2} \cdot$ Sadrollah Motamed ${ }^{2} \cdot$ Abdolreza Rouientan $^{2} \cdot$ Naser Mozafari $^{2} \cdot$ Soraya Shahrokh $^{2}$. \\ Shahin Mohammadsadeghi' ${ }^{2} \cdot$ Feyzollah $\mathrm{Niazi}^{2} \cdot \mathrm{Khalil} \mathrm{Rostami}^{2}$
}

Received: 20 January 2021 / Accepted: 3 May 2021 / Published online: 3 June 2021

(c) The Author(s), under exclusive licence to Springer-Verlag GmbH Germany, part of Springer Nature 2021

\begin{abstract}
Background On March 11, 2020, the World Health Organization (WHO) announced the COVID-19 outbreak as a new pandemic. In the meantime, plastic surgeons postponed their appointments due to the fair and rational allocation of medical supplies. These limitations made all junior and senior residents perform operations only on traumatic patients rather than those needing reconstructive procedures. This study aims to determine the effects of the COVID-19 pandemic on plastic surgery training programs in Iran. Also, the number of canceled surgeries will be determined to see the effects of the pandemic on the patients.

Methods This retrospective case study considers a six-month timeframe in two consecutive years before and after the COVID-19 pandemic. For this purpose, the researchers evaluated the number of surgeries, types of procedures, age distribution, and gender distribution. The training program data of plastic surgery residents were collected from their logbooks and then analyzed in IBM SPSS Statistics 26. Differences were considered significant if $p<0.05$ at a $95 \%$ confidence level. Results The total number of surgeries decreased by $23.5 \%$ after the COVID-19 outbreak $(p<0.05)$. There was a $29.9 \%$ reduction in trauma cases, $-78.9 \%$ in aesthetic surgeries, $-17.7 \%$ in reconstructive surgeries, $-51.8 \%$ in craniofacial surgeries, and $-59.5 \%$ in microscopic surgeries for each resident.

Conclusions This study provides an insight into the severity of the pandemic effects on the plastic surgery training programs and the patients. The reduced number of surgeries led to a depletion in surgical skills training. These effects will not wear off immediately after the pandemic; therefore, it is necessary to observe whether the pandemic will have any lasting effects on this subspecialty.

Level of evidence: Level IV, risk/prognostic study
\end{abstract}

Keywords COVID-19 · Aesthetic surgery · Plastic surgery $\cdot$ Residency program

\section{Introduction}

Wuhan Municipal Health Commission, China, reported a cluster of cases of pneumonia caused by a novel virus called SARS-CoV2 on December 31, 2019 [1]. The affected

Abdoljalil Kalantar-Hormozi

kalantarj@yahoo.com

1 Research and Development Centre, School of Medicine, Panzdah-E Khordad Teaching Hospital, Shahid Beheshti University of Medical Sciences, Tehran, Iran

2 Department of Plastic Surgery, School of Medicine, Panzdah-E Khordad Teaching Hospital, Shahid Beheshti University of Medical Sciences, Tehran, Iran patients manifested a wide range of symptoms from asymptomatic to severe acute respiratory syndrome and acute thromboembolic diseases. On March 11, 2020, the WHO announced the COVID19 outbreak as a new pandemic [2]. Ever since, all countries worldwide have undergone lockdowns of varying durations and limitations. Most medical subspecialties adjusted their activities to face this new situation, considering ethical principles and values [3]. In the meantime, plastic surgeons have postponed their appointments due to the fair and rational allocation of medical supplies. In Iran, the first case of the COVID-19 was reported on February 19, 2020. According to Iranian health authorities, there were 59,899 COVID-19 deaths in Iran with more than 1,615,184 confirmed active cases until February 20, 
2021. After the first case was diagnosed in Iran, the Ministry of Health and Medical Education decided to cancel all elective surgeries for about a month. Nearly two and a half months later, the Iranian government lifted the limitations and allowed people to go back to work [4] including a limited number of elective surgeries under specific conditions. All these on and off limitations during the last year affected patients as well as health organizations worldwide [5]. Postponing non-delayable surgeries may harm the patient health and life quality [6-8]. Moreover, a very long waiting list is now being made for the foreseeable future in which patients should be prioritized with respect to their clinical status. This prioritization process also delays the surgeries of some patients with benign but potentially malignant situations.

Aesthetic and reconstructive surgeries are among of the most popular subspecialties among trainees. This field needs high levels of skills in different types of surgeries. Aesthetic and reconstructive surgery residents need high scores and consultants' confirmation on their skills to be board-certified. This makes residents show competence in performing a wide range of index operating procedures, being involved in teaching, and improving their abilities to manage multiple situations and leadership roles [9]. With the advent of SARS-CoV2 in the late 2019, Iran's Ministry of Health and Medical Education decided to cancel and postpone elective surgeries, something which played a significant role in aesthetic and reconstructive surgeries. These limitations made all junior and senior residents only operate on traumatic patients rather than aesthetic and reconstructive ones.

The history of the contemporary plastic surgery in Iran dates back to 70 years ago. However, there are now only three plastic and reconstructive surgery subspecialty hospitals in Tehran which admit patients from all over Iran for medical care. Moreover, in other big cities of Iran, there are small plastic surgery wards that cannot provide wide ranges of treatment due to the lack of resources. As a result, those three subspecialty hospitals are considered the main centres for treating plastic surgery patients. One of these centres, in which this study was conducted, is panzdah-e Khordad Teaching Hospital. Annually, 5 or 6 general surgeons start their plastic surgery residency programs in this hospital. Being a resident in this tertiary referral centre in a country like Iran and operating on hundreds of cases will make very successful surgeons. This pandemic has affected multiple levels of education and medical care including plastic surgery resident skills.

There is still no exact data regarding the number of cancellations due to either a heavy workload or a lack of reliable databases in most countries [10,11]. Collecting nearly accurate data on the number of surgeries that have been canceled or postponed can help develop certain protocols to cope with the pandemic and its subsequent outcomes in order to confront a long waiting list and affected training programs of residents [12]. This study aims to determine the effects of the COVID-19 on plastic surgery training programs in a tertiary referral centre in Iran. Also, as the secondary aim of the study, the number of canceled surgeries will be determined to see the effects of the pandemic on the patients.

\section{Materials and methods}

In this retrospective case study, a six-month timeframe was considered in two consecutive years before and after the COVID-19 pandemic. The pre-pandemic period was from March 20 to September 21, 2019, whereas the postpandemic period was from March 20 to September 21, 2020. The total numbers of admissions for surgeries were evaluated and compared within the two above periods. Furthermore, the types of procedures (plastic and aesthetic, reconstructive, microscopic, craniofacial, and traumatic) were evaluated as well as age and the gender distribution. Burn patients were classified as the reconstructive category. The data were collected from the hospital archive and patient files, whereas the information of the plastic surgery training program was obtained from the logbooks. After the raw data were sorted into time and surgery type subgroups, the frequency charts were drawn in IBM SPSS Statistics 26 to calculate significant statistics. Differences were considered statistically significant if $p<0.05$ at a $95 \%$ confidence level.

\section{Residency program}

To become a plastic surgeon in Iran, medical doctors must pass a four-year general surgery program and then try to enter the subspecialty with a high score and sufficient experience in general surgery. The plastic surgery residency program lasts three years. In the first year, residents learn how to manage emergency departments and surgeries under their senior resident's supervision. Therefore, all the traumatic surgeries of the maxillofacial, head and neck, and upper and lower extremities are performed by the first-year residents including microscopic traumatic surgeries. In the second year, they partake in reconstructive and elective microscopic surgeries along with third-year residents under the supervision of consultants. Third-year residents operate on aesthetic and craniofacial patients as well as sophisticated cases.

This hospital accepts five or six plastic surgery residents and one or two hand surgery fellowships annually. Considering the large number of patient admissions and the lack of even distributions, ED admissions were 70 patients on average before the pandemic. Seven operating theatres were used, and 67 OR personnel covered the shifts during prepandemic period. 
Table 1 Exact numbers of each subgroup in pre- and post-pandemic periods

\begin{tabular}{|c|c|c|c|c|}
\hline \multicolumn{5}{|c|}{ Year * surgery cross tabulation } \\
\hline & & \multicolumn{2}{|l|}{ Year } & \multirow[t]{2}{*}{ Total } \\
\hline & & 2019 & 2020 & \\
\hline \multirow[t]{8}{*}{ Surgery } & Aesthetic s & 314 & 66 & 380 \\
\hline & Reconstructive $\mathrm{s}$ & 685 & 562 & 1247 \\
\hline & Craniofacial s & 150 & 74 & 224 \\
\hline & Upper extremities trauma & 4233 & 3476 & 7709 \\
\hline & Lower extremities trauma & 299 & 292 & 591 \\
\hline & Maxillofacial trauma & 786 & 574 & 1360 \\
\hline & Microscopic s & 234 & 92 & 326 \\
\hline & Amputation & 194 & 163 & 357 \\
\hline Total & & 6895 & 5299 & 12,194 \\
\hline
\end{tabular}

\section{Results}

Out of 7027 patients admitted during the pre-pandemic period, 132 patients refused to undergo their surgeries. A total number of 6895 operations were performed in five subgroups, i.e. plastic surgery, reconstructive surgery, craniofacial surgery, microscopic surgery, and traumatic surgery. In the 2020 post-pandemic timeframe, 5371 patients were admitted. Among them, 72 surgeries were canceled due to either personal issues or clinical situations.

Table 1 presents the details about the number of patients who underwent each surgery in 2019 and 2020. Regarding the mean number of surgeries per month in 2019 , there were 53 plastic surgeries, 114 reconstructive surgeries, 25 craniofacial surgeries, 38 microscopic surgeries, and 295 traumatic surgeries. These numbers in 2020 were 11 in plastic (-79.2\%), 104 in reconstructive $(-8.7 \%), 12$ in craniofacial (-52\%), 16 in microscopic surgery (-57.8\%), and 269 in traumatic surgery $(-8.8 \%)$. In the pre-pandemic period, the mean patient age was 28.91 years (youngest 0 , oldest 92, with an SD of 16.41), whereas it was 29.22 years in the post-pandemic period (youngest 0 , oldest 95, with an SD of 15.25). In 2019, $75.7 \%$ of patients were male (5322 patients), and $24.3 \%$ were female (1705 patients). In $2020,80.6 \%$ of them were male (4331 patients), whereas $19.4 \%$ were female (1040 patients). Tables 2 and 3 report the gender distribution for subgroups in 2019 and 2020, respectively. Moreover, Figs. 1 and 2 demonstrate the data about age distribution.

In 2019, there were seven first-year, six second-year, and six third-year residents in the designated hospital. Moreover, there were five first-year, seven second-year, and six third-year residents in 2020. Out of the seven operating theatre of the hospital, five were alternatively active and inactive during the pandemic, and there was a $20 \%$ reduction in the number of OR personnel. As discussed in Materials and methods section, each first-year resident operated on 918 traumatic patients in 2019, whereas they experienced 643 surgeries of the same type in 2020 (a $29.9 \%$ decrease). Figure 3 shows the details regarding the number of surgeries per resident. The reduction rates of in other fields were reported as $-78.9 \%$ in aesthetic surgeries, $-17.7 \%$ in reconstructive surgeries, $-51.8 \%$ in craniofacial surgeries, and $-59.5 \%$ in microscopic surgeries.

\section{Discussion}

This study provides an insight into the severity of effects left by the COVID-19 pandemic on plastic surgery training programs and patients by comparing the pre- and post-pandemic timeframes. Elective surgeries were

Table 2 Gender distribution 2019

\begin{tabular}{|c|c|c|c|c|c|c|c|}
\hline \multicolumn{8}{|c|}{ Gender * surgery cross tabulation 2019} \\
\hline & & \multicolumn{4}{|l|}{ Gender } & \multirow{2}{*}{\multicolumn{2}{|c|}{ Total }} \\
\hline & & \multicolumn{2}{|l|}{ Male } & \multicolumn{2}{|c|}{ Female } & & \\
\hline & & Count & $\%$ within gender & Count & $\%$ within gender & Count & $\%$ within gender \\
\hline \multirow[t]{8}{*}{ Surgery } & Plastic s & 78 & $1.5 \%$ & 236 & $14.2 \%$ & 314 & $4.6 \%$ \\
\hline & Reconstructive s & 484 & $9.3 \%$ & 201 & $12.1 \%$ & 685 & $9.9 \%$ \\
\hline & Craniofacial s & 81 & $1.5 \%$ & 69 & $4.1 \%$ & 150 & $2.2 \%$ \\
\hline & Upper extremities trauma & 3575 & $68.4 \%$ & 658 & $39.5 \%$ & 4233 & $61.4 \%$ \\
\hline & Lower extremities trauma & 233 & $4.5 \%$ & 66 & $4.0 \%$ & 299 & $4.3 \%$ \\
\hline & Maxillofacial trauma & 464 & $8.9 \%$ & 322 & $19.3 \%$ & 786 & $11.4 \%$ \\
\hline & Microscopic s & 148 & $2.8 \%$ & 86 & $5.2 \%$ & 234 & $3.4 \%$ \\
\hline & Amputation & 165 & $3.2 \%$ & 29 & $1.7 \%$ & 194 & $2.8 \%$ \\
\hline \multicolumn{2}{|l|}{ Total } & 5228 & $100.0 \%$ & 1667 & $100.0 \%$ & 6895 & $100.0 \%$ \\
\hline
\end{tabular}


Table 3 Gender distribution 2020

\begin{tabular}{|c|c|c|c|c|c|c|c|}
\hline \multicolumn{8}{|c|}{ Gender * surgery cross tabulation 2020} \\
\hline & & \multicolumn{4}{|c|}{ Gender } & \multirow{2}{*}{\multicolumn{2}{|c|}{ Total }} \\
\hline & & \multicolumn{2}{|l|}{ Male } & \multicolumn{2}{|c|}{ Female } & & \\
\hline & & Count & $\%$ within gender & Count & $\%$ within gender & Count & $\%$ within gender \\
\hline \multirow[t]{8}{*}{ Surgery } & Plastic s & 19 & $0.4 \%$ & 47 & $4.6 \%$ & 66 & $1.2 \%$ \\
\hline & Reconstructive s & 436 & $10.2 \%$ & 126 & $12.3 \%$ & 562 & $10.6 \%$ \\
\hline & Craniofacial s & 26 & $0.6 \%$ & 48 & $4.7 \%$ & 74 & $1.4 \%$ \\
\hline & Upper extremities trauma & 2994 & $70.0 \%$ & 482 & $47.2 \%$ & 3476 & $65.6 \%$ \\
\hline & Lower extremities trauma & 224 & $5.2 \%$ & 68 & $6.7 \%$ & 292 & $5.5 \%$ \\
\hline & Maxillofacial trauma & 380 & $8.9 \%$ & 194 & $19.0 \%$ & 574 & $10.8 \%$ \\
\hline & Microscopic s & 61 & $1.4 \%$ & 31 & $3.0 \%$ & 92 & $1.7 \%$ \\
\hline & Amputation & 137 & $3.2 \%$ & 26 & $2.5 \%$ & 163 & $3.1 \%$ \\
\hline Total & & 4277 & $100.0 \%$ & 1022 & $100.0 \%$ & 5299 & $100.0 \%$ \\
\hline
\end{tabular}

forbidden for several months in the post-pandemic timeframe, whereas surgeons could only perform non-delayable surgeries in other months. As discussed earlier, there are only three plastic surgery subspecialty hospitals in Iran as well as some plastic surgery wards of the large cities which suffer from lack of resources. This uneven distribution makes the three hospitals the main plastic surgery centres that admit a plethora of patients either for traumatic surgeries or elective ones. Thus, even with on and off limitations, there has been a significant number of operations during the pandemic. The total number of surgeries decreased by $23.5 \%$ during the post-pandemic period $(\mathrm{p}<0.05)$. These limitations have also affected the residency training programs. The number of patients has reached half and below that of the last year in plastic, craniofacial, and microscopic surgeries. In other words, residents had half the chance to improve their skills in this field when their time was flying. Although traumatic surgeries lasted during the post-pandemic period, there was a $29.9 \%$ reduction in every resident's cases. In reconstructive surgeries, there was an $8.7 \%$ decrease in the total number of cases which caused a $17.7 \%$ reduction in resident cases. The reduced number of surgeries would lead to a depletion in surgical skills training, and reduced discussion contents in morning meetings and teaching conferences. Furthermore, if residents fail to complete their logbooks by the graduation time, they will miss their board certification exams. These effects will not

Fig. 1 Age distribution 2019

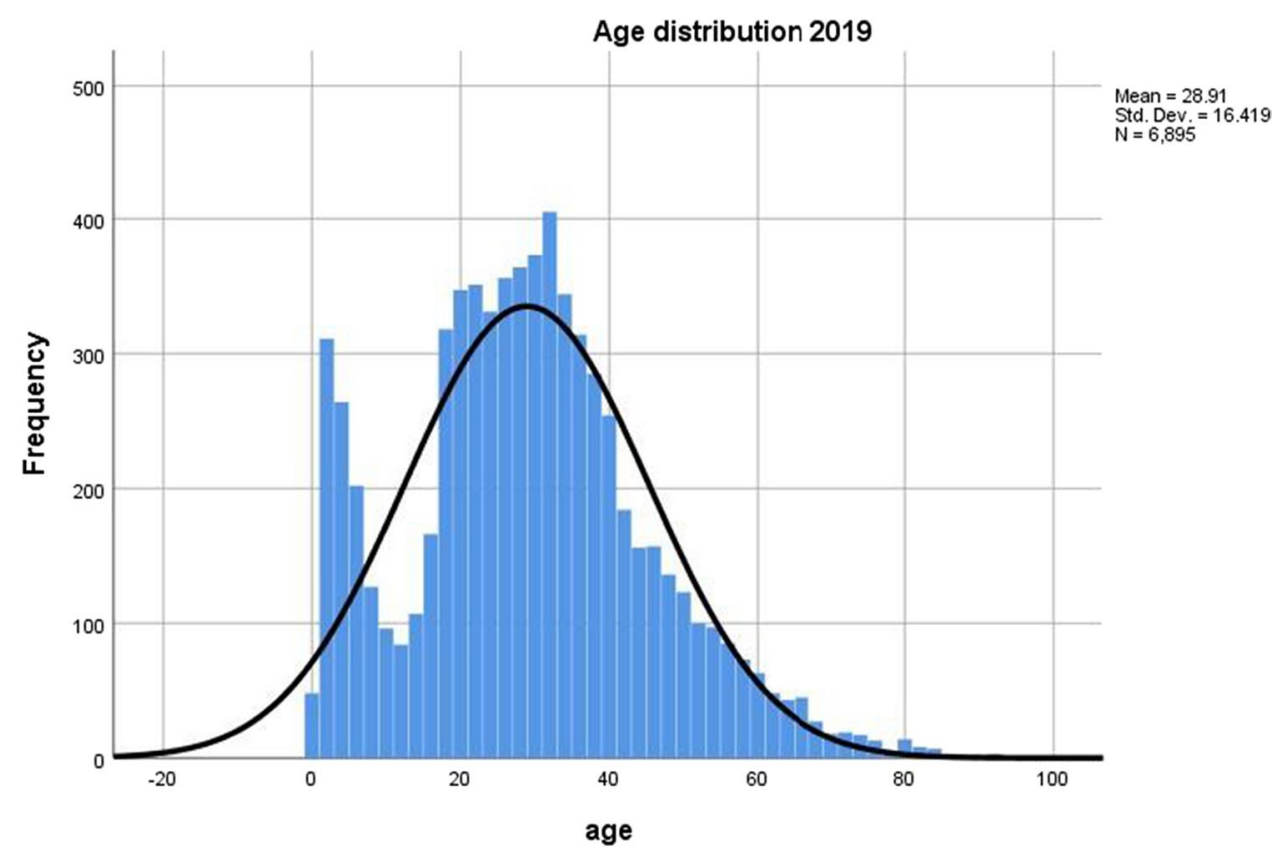


Fig. 2 Age distribution 2020

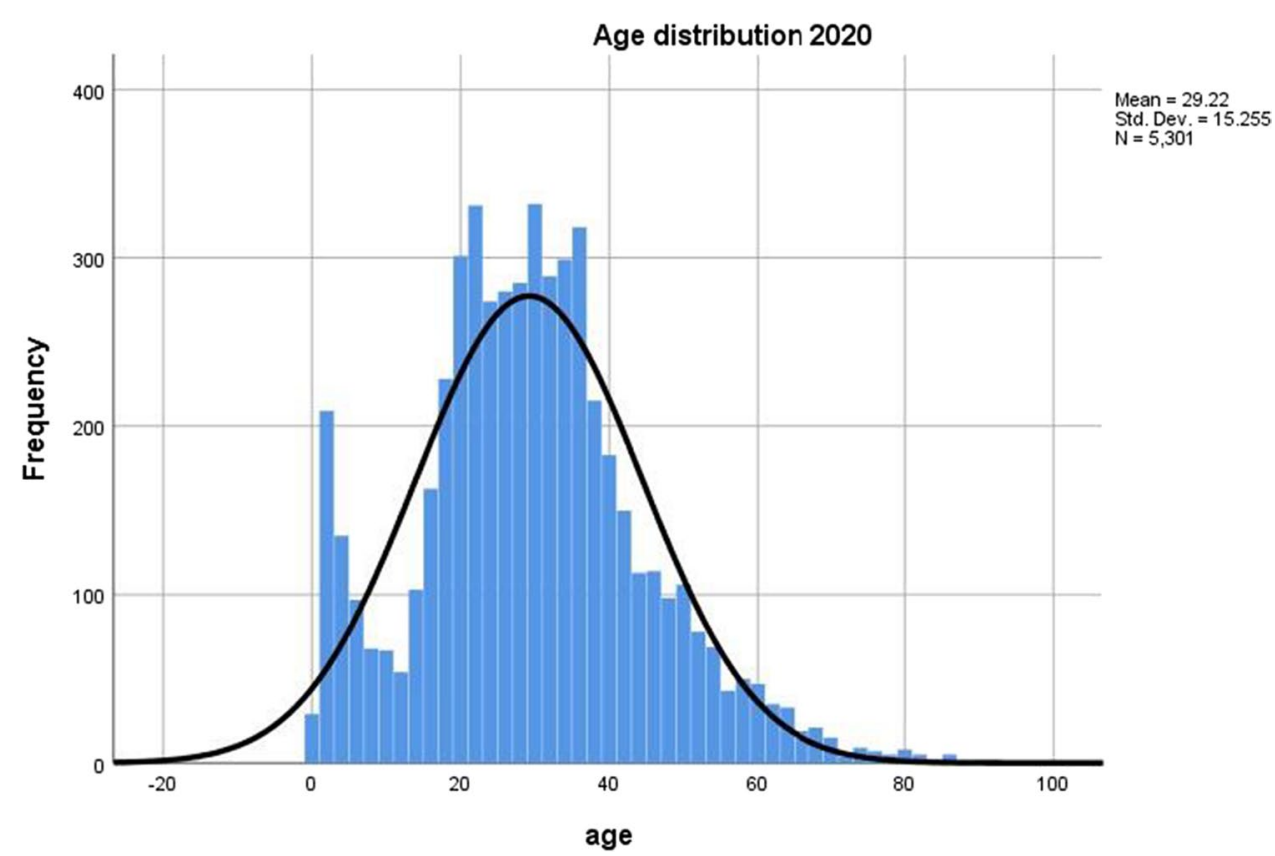

stop immediately after the pandemic. It is also necessary to determine whether this pandemic will have any lasting effects on this subspecialty. Conducting a survey on the effects of this pandemic on plastic surgery in Brazil, Vitor Penteado et al. concluded that reconstructive surgeries were the only survival cause of plastic surgery training programs during this disaster [13]. In India, Sangeeta Thakurani et al. studied this specialty during the pandemic. They concluded that the escalating surge of plastic surgery cases in India was hit hard by the COVID-19 pandemic and that it would take a long time and serious actions to return to normalcy [14]. There are also several studies addressing the effects of this pandemic on variable specialties. In nearly all of them, residency training programs have been reported to be the most affected area [15-17]. The use of telemedicine has increased during this pandemic; however, some countries suffer from the lack of required infrastructures.

In the designated hospital, the first screening step during the pandemic is temperature screening before patients are admitted from ED. Suspicious patients are led to the grey ward in which a physician will examine them for clinical evaluation. An operating theatre then is prepared for the suspicious patients who need emergency surgeries. The hospital also employs educated personnel who will provide sufficient care for these patients both before and after their operations. They are also provided with personal protective equipment (PPE) including coveralls (a one-piece suit), masks, and
Fig. 3 Number of surgeries per residents in 2019 and 2020

\section{Number of surgeries per residents in 2019 and}

\section{0}

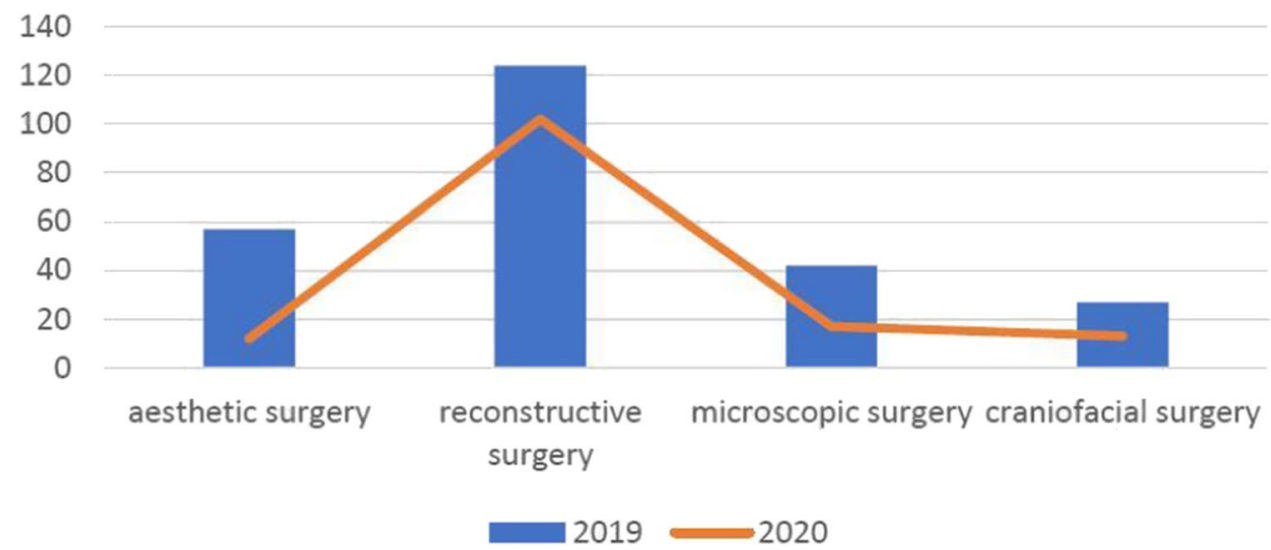


goggles. The suspicious patients with non-emergency situations would be referred to a COVID-19 centre for further evaluation. Their surgical operations would be scheduled if the COVID-19 centre grants the permission. In elective surgeries, patients are screened with PCR tests. Surgeries are canceled in positive patients. Despite the strict precautions for prevention, one-third of operating theatre personnel were infected during the post-pandemic period.

In the early days of the pandemic when there was insufficient data about this novel virus, the global notion was that the virus will mostly affect the elderly. Therefore, the authors of this study decided to consider differences in age distribution before and after the pandemic to evaluate the pandemic effects on the admitted patients (to see whether this notion caused less hospital admissions or not). There were no significant differences in age distribution before and after the pandemic.

It is also necessary to consider the effects of these limitations on patients. There are several conditions in which there is a golden opportunity for an intervention which can yield good outcomes. The lockdown may cause a waste of time for these patients. In addition, a very long waiting list is made for the post-pandemic period when patients must be prioritized in terms of their clinical conditions. This means a longer postponement for some patients, something which might be dangerous and affect their quality of life [6-8]. According to Negopdiev et al., it is also essential to increase the workload to shorten the time required to return to normalcy. They discussed a global estimation of elective surgery cancellation and estimated whether countries would increase their surgery cases by $20 \%$. They also stated that it would take nearly 45 weeks to return to normal workloads and waiting lists [18].

According to the results, some interventions must be performed by healthcare providers in order to minimize the harm done to patients and residents during the pandemic. Here are some educational intervention examples:

1. Documenting and recording rare cases and surgeries performed during the pandemic, making them available to all residents, and using webinars to discuss those surgeries so that no one misses them.

2. Holding virtual morning meetings and allowing everyone to participate in these meetings. This intervention needs suitable infrastructures.

3. Using webinars rather than conventional in-person classes.

4. Explaining cascade training to residents so that new residents, who do not have access to their field's prominent professors due to the pandemic, do not miss out on education.
Here are some examples of interventions to decrease patients' harm:

1. Senior residents of each field can review patients' files and sort them according to their clinical situation to ensure that no patient is missed due to a long waiting list, especially for non-delayable surgeries.

2. Reducing hospital visits by using telemedicine services for non-urgent and follow-up appointments.

3. Implementing screening protocols for all patients, as well as specific treatment protocols and facilities for COVID19 patients who require surgery.

4. Altering hospitals' hardware to admit patients during the pandemic; for example, designing suitable waiting rooms with appropriate ventilation that are adequate for social distancing, admitting a maximum of one or two patients in an ordinary $4-6$ bed-rooms and providing good ventilation in all rooms, allocating specific facilities for suspicious patients and using the rest of equipment for other patients for elective surgeries; allocating separate facilities for ED patients and expediting their treatment process.

\section{Conclusions}

It is impossible to reverse the pandemic effects on our lives and activities; however, it is possible to adapt everything to the new situation to sustain less damage during the pandemic. Healthcare providers can perform some interventions to reduce the negative effects on patients and residents. These interventions include using webinars instead of conventional in-person classes, recording the limited operations performed during the pandemic to educate residents who missed them, adjusting operating theatres and waiting rooms in clinics in order to comply with social distancing protocols, and screening patients at the time of admission in addition to isolating them for a specific interval before surgery. Making these changes in teaching hospitals can slightly increase the admission rates during the pandemic. This study provides an insight into what the world will face after the pandemic, how much increase is expected in the workload, and how long it will take to return to normalcy. The next important action that healthcare providers must take in this journey is to define new protocols to admit patients during the pandemic and after that.

Acknowledgements panzdah-e Khordad Research Centre, Shahid Beheshti University of Medical Sciences, Tehran, Iran 


\section{Declarations}

Ethics approval All procedures performed in studies involving human participants were in accordance with the ethical standards of the institutional and/or national research committee and with the 1964 Helsinki Declaration and its later amendments or comparable ethical standards. This is an observational study that was approved by Ethics Committee of Shahid Beheshti University of Medical Sciences under the ethical code of IR.SBMU.RETECH.REC.1399.836.

Patient consent Patients signed informed consent regarding publishing their data.

Informed consent Informed consent was obtained from all individual participants included in the study.

Conflict of interest The authors Abdoljalil Kalantar-Hormozi, Zahra Habibzadeh, Masoud Yavari, Seyed Mehdi Mousavizadeh, Seyed Esmaeil Hassanpour, Sadrollah Motamed, Abdolreza Rouientan, Naser Mozafari, Soraya Shahrokh, Shahin Mohammadsadeghi, Feyzollah Niazi, Khalil Rostami declare no conflict of interest.

\section{References}

1. Wang C, Horby PW, Hayden FG, Gao GF (2020) A novel coronavirus outbreak of global health concern. Lancet 395(10223):470-473

2. World Health Organization (2020) WHO Director-General's opening remarks at the media briefing on COVID-19. https:// www.who.int/director-general/speeches/detail/who-director-gener al-s-opening-remarks-at-the-media-briefing-on-covid-19---11march-2020. Accessed December 2020

3. Kirkpatrick JN, Hull SC, Fedson S, Mullen B, Goodlin SJ (2020) Scarce-resource allocation and patient triage during the COVID19 pandemic: JACC Review Topic of the Week. JACC CardioOncol 76(1):85-92

4. Sharifi H, Jahani Y, Mirzazadeh A, Gohari MA, Nakhaeizadeh M, Shokoohi M, et al (2020) Estimating the number of COVID19-related infections, deaths and hospitalizations in Iran under different physical distancing and isolation scenarios. Int J Health Policy Manag. https://doi.org/10.34172/ijhpm.2020.134

5. Søreide K, Hallet J, Matthews JB, Schnitzbauer AA, Line PD, Lai $P$, et al (2020) Immediate and long-term impact of the COVID-19 pandemic on delivery of surgical services. Br J Surg 107(10):1250-1261

6. Grass F, Behm KT, Duchalais E, Crippa J, Spears GM, Harmsen WS et al (2020) Impact of delay to surgery on survival in stage I-III colon cancer. Eur J Surg Oncol 46(3):455-461
7. Kompelli AR, Li H, Neskey DM (2019) Impact of delay in treatment initiation on overall survival in laryngeal cancers. OTO Open 160(4):651-657

8. Shin DW, Cho J, Kim SY, Guallar E, Hwang SS, Cho B et al (2013) Delay to curative surgery greater than 12 weeks is associated with increased mortality in patients with colorectal and breast cancer but not lung or thyroid cancer. Ann Surg Oncol 20(8):2468-2476

9. Zuo KJ, Retrouvey H, Wanzel KR (2019) Factors that affect medical students' perception and impression of a plastic surgery program: the role of elective rotations and interviews. Ann Plast Surg 82(2):224-228

10. Spinelli A, Pellino G (2020) COVID-19 pandemic: perspectives on an unfolding crisis. Br J Surg 107(7):785-787

11. Tuech J-J, Gangloff A, Schwarz L (2020) Our challenge is to adapt the organization of our system to the six stages of the epidemic to go beyond the COVID-19 crisis. Br J Surg 107(7):e189

12. Ozturk CN, Kuruoglu D, Ozturk C, Rampazzo A, Gurunian R (2020) Plastic surgery and the COVID-19 pandemic: a review of clinical guidelines. Ann Plast Surg 85(2S Suppl 2):S155-S160

13. Pagotto VPF, Abbas L, Goldenberg DC, Lobato RC, do Nascimento BB, Monteiro GGR et al (2020) The impact of COVID-19 on the plastic surgery activity in a high-complexity university hospital in Brazil: the importance of reconstructive plastic surgery during the pandemic. Eur J Plast Surg. 43(6):819-24

14. Thakurani S, Gupta S (2021) Evolution of aesthetic surgery in India, current practice scenario, and anticipated post-COVID-19 changes: a survey-based analysis. Eur J Plast Surg 44(1):129-138

15. Abedi AR, Aliakbari F, Ghiasy S, Allameh F, Ghanbari MA (2020) The Impact of the First Covid 19 Pandemic on Urology Residency Training in Iran. Men's Health J 4(1):e21-e

16. Patel NM, Khajuria A, Khajuria A (2020) Utility of a webinar to educate trainees on UK core surgical training (CST) selection-A cross sectional study and future implications amidst the COVID19 pandemic. Ann Med Surg (Lond) 59:35-40

17. Khan H, Williamson M, Trompeter A (2021) The impact of the COVID-19 pandemic on orthopaedic services and training in the UK. Eur J Orthop Surg Traumatol 31(1):105-109

18. Negopdiev D, Collaborative C, Hoste E (2020) Elective surgery cancellations due to the COVID-19 pandemic: global predictive modelling to inform surgical recovery plans. Br J Surg 107(11):1440-1449

Publisher's note Springer Nature remains neutral with regard to jurisdictional claims in published maps and institutional affiliations. 